\title{
Abundance of reactive oxygen species (ROS) is associated with tumor aggressiveness, immune response and worse survival in breast cancer
}

Masanori oshi

Roswell Park Comprehensive Cancer Center

Shipra Gandhi

Roswell Park Comprehensive Cancer Center

Li Yan

Roswell Park Comprehensive Cancer Center

Yoshihisa Tokumaru

Roswell Park Comprehensive Cancer Center

Rongrong Wu

Roswell Park Comprehensive Cancer Center

Akimitsu Yamada

Yokohama City University Hospital: Yokohama Shiritsu Daigaku Fuzoku Byoin

\section{Ryusei Matsuyama}

Yokohama City University Hospital: Yokohama Shiritsu Daigaku Fuzoku Byoin

Itaru Endo

Yokohama City University Hospital: Yokohama Shiritsu Daigaku Fuzoku Byoin

Kazuaki Takabe ( $\nabla$ kazuaki.takabe@roswellpark.org )

Roswell Park Comprehensive Cancer Center https://orcid.org/0000-0002-6435-4241

\section{Research Article}

Keywords: biomarker, GSVA, reactive oxygen species, survival analysis, tumor immune microenvironment.

Posted Date: February 24th, 2022

DOI: https://doi.org/10.21203/rs.3.rs-1375563/v1

License: (c) (i) This work is licensed under a Creative Commons Attribution 4.0 International License.

Read Full License 


\section{Abstract}

Purpose: Reactive oxygen species (ROS) are oxygen-containing molecules that have high reactivity and play roles in protection or harm the cancer cells. We aimed to clarify the clinical relevance of ROS in breast cancer (BC) tumor microenvironment (TME). We hypothesized that it is associated with worse BC patient outcomes.

Methods: ROS score was generated by Gene Set Variation Analysis of Hallmark ROS pathway gene set and a total of $6,245 \mathrm{BC}$ patients were analyzed.

Results: High ROS BC significantly enriched cell proliferation-related gene sets (MYC targets v1 and v2, G2M checkpoint, E2F targets), pro-cancer-related gene sets (DNA repair, unfolded protein response, MTORC1 signaling, PI3K/AKT/MTOR signaling, glycolysis, and oxidative phosphorylation), immunerelated gene sets (inflammatory response, allograft rejection, interferon- $\alpha$ and $\gamma$ responses, complement, and IL6/JAK/STAT3 signaling), and infiltrated immune cells (CD4 ${ }^{+}$memory and CD $8^{+}$T cells, Th1 and Th2, dendritic cells, Tregs, M1 and M2 macrophages) and B-cells, as well as elevated cytolytic activity consistently in both METABRIC and GSE96058 cohorts. Cancer cells were the major source of ROS in BC TME of single cell sequence (GSE75688) cohort. High ROS was associated with intratumor heterogeneity, homologous recombination defects, mutation rates and neoantigens, and with clinical aggressiveness in AJCC stage, Nottingham grade and Ki67 expression, as well as worse overall survival in both GSE96058 and METABRIC, and with worse disease-specific survival in METABRIC.

Conclusion: Abundant ROS in BC patients is associated with abundant mutations, aggressive cancer biology, immune response and worse survival.

\section{Introduction}

The body maintains its homeostasis and health by balancing oxidative system that produce energy and antioxidant system that prevent the negative effects of excess oxidation, also known as oxidative stress $[1,2]$. Recently, it has become clear that excessive oxidation and oxidative stress directly damages DNA and are deeply involved not only in carcinogenesis but also in cancer progression, including cancer growth, invasion, and metastatic spread.

Reactive oxygen species (ROS) are oxygen-containing molecules that have high reactivity. Hydroxyl $\left(\mathrm{OH}^{\star}\right)$ and superoxide $\left(\mathrm{O}_{2}{ }^{*}\right)$ free radicals both comprise ROS along with hydrogen peroxide $\left(\mathrm{H}_{2} \mathrm{O}_{2}\right)$. ROS are physiologically produced in the mitochondrial electron-transfer system, peroxisomes and phagosomes in the cells, where they contribute to energy production and phagocytosis [3]. ROS is also produced in small amounts by the NADPH oxidase (Nox) family at the plasma membrane and is involved in a number of intracellular signaling pathways, such as Ras, c-jun N-terminal kinase (JNK), p38 and mitogen-activated protein kinase (MAPK), and PI3K/AKT/mTOR pathways. Recently, it has been shown that ROS is involved in cell proliferation [4,5] as well as autophagy [6], apoptosis [7], and inflammation via the NLRP3 inflammasome and nuclear factor-kB (NF-kB) pathway [8, 9]. Cells experience oxidative stress if either the 
ROS production increases or the number of scavenged ROS decreases [10]. While ROS have physiological functions as described, they can be harmful by directly damaging DNA during oxidative stress. Our group and others have shown that abundant DNA damage cause higher mutation load that leads to carcinogenesis and aggravation of cancer [11]. Ongoing aerobic glycolysis followed by pyruvate oxidation in the mitochondria (the Warburg effect) generates ROS which in turn increases the activity of oncogenes and receptors. This results in stimulation of oxidizing enzymes and/or growth factordependent pathways, thus inducing instability of genes [10]. For this reason, cells are equipped with antioxidant system that either take in antioxidants or produce them by enzymes. Although excessive induction of oxidative stress is expected to prevent and/or treat cancer since cancer cells have higher ROS than normal cells as a base line, ROS may aggravate cancer by promoting cell proliferation. To this end, the clinical relevance of abundant ROS in breast cancer remains unclear.

In this study, we generated a ROS score using ROS-related gene expressions in human breast cancer to quantify the abundance of ROS and investigated its clinical relevance. Gene Set Variation Analysis (GSVA) with 200 ROS-related genes defined by Molecular Signatures Database (MSigDB) Hallmark collection was used to generate the ROS score [12]. This approach is commonly used in genetic research because pathways involve various genes, and a single gene expression may fail to grasp the whole picture. For instance, we found the association between G2M checkpoint pathway and pancreatic cancer survival and drug response $[13,14]$, angiogenesis and aggressiveness of gastric cancer and worse survival [15], and DNA repair signaling [16] as well as unfolded protein response [17] and worse survival in liver cancer. We have demonstrated in breast tumor that pathways such as early estrogen response [18], KRAS signaling [19], angiogenesis [20], inflammation [21], thermogenesis [22], adipogenesis [23], and apoptosis [24] are associated with survival, and G2M checkpoint pathway [25] and E2F pathway [26] predict response to neoadjuvant chemotherapy in Luminal A subtype. In this study, we hypothesized that abundant ROS is significantly associated with cancer aggressiveness and tumor microenvironment, as well as patient survival.

\section{Materials And Methods}

\section{Data acquisition}

Clinical information and gene expression were obtained on 1,903 breast cancer patients from the Molecular Taxonomy of Breast Cancer International Consortium (METABRIC) cohort, as we previously reported $[27,28]$. The GSE96058 cohort consists of full transcriptome profile of resected tumors from 3,273 breast cancer patients whose clinical data was obtained from the Swedish Breast Cancer Analysis Network (SCAN-B) [29]. Transcriptomic data was also obtained on 1,069 female breast cancer patients from The Cancer Genome Atlas (TCGA) cohort [30]. The GSE75688 cohort has single-cell RNAsequencing data of cancer cells, myeloid cells, immune cells, and stromal cells in breast cancer [31], which was obtained from Gene Expression Omnibus.

\section{ROS signaling score}


Liberzon et al. reported the Molecular Signatures Database (MSigDB) hallmark gene set collection, one of the most comprehensive and widely used databases of gene sets for performing gene set enrichment analysis [12]. We used "HALLMARK REACTIVE OXYGENE SPECIES" gene sets, that was calculated by Gene Set Variation Analysis (GSVA) algorithm [32], in the similar manner as our previous reports on KRAS signaling [19], G2M checkpoint [13, 14, 25], E2F targets [26], angiogenesis [15], estrogen response [18], inflammation [21], thermogenesis [22], adipogenesis [23], and apoptosis [24].

\section{Other scores}

Cytolytic activity (CYT) score estimates the overall immune activity by expression pattern of granzyme A (GZMA) and perforin (PRF1) genes [33-37]. xCell scores were used as immune cells score calculating by the xCell algorithm [38], as we previously reported [39-41]. Score results provided by Thorsson et al. [42] in the TCGA cohort were utilized to calculate homologous recombination defects (HRD), intratumor heterogeneity, single nucleotide variation (SNV), silent and non-silent mutation rate, and fraction altered.

\section{Gene set enrichment analysis}

The difference in activated signaling pathways between low- and high-ROS score groups were investigated by Gene Set Enrichment Analysis (GSEA) [43] using GSEA Java software (version 4.2) with MSigDB Hallmark gene sets [12]. False discovery rate (FDR) $<25 \%$ was defined as statistically significant, as recommended by GSEA.

\section{Other statistical analyses}

All analyses and data plots were generated using Microsoft Excel (version 16) and R software (version 4.0.1). The analysis of the comparison of groups used the Kruskal-Wallis test, the Mann-Whitney $U$ test, or the Fisher exact test. Log-rank test and the Kaplan-Meier plot were used for survival analyses. Values of $p$ $<0.05$ generally indicate a statistically significant difference.

\section{Results}

Breast cancer with a high reactive oxygen species (ROS) score enriched cell proliferation and other procancerous gene sets

The Gene set variation analysis (GSVA) algorithm on MSigDB was used to define the ROS score (Table S1), similar to how we defined the other scores in our previous publications [13-16]. We first investigated the underlying mechanism involved in the score by conducting Gene Set Enrichment Analysis (GSEA) on two independent breast cancer cohorts, GSE96058 and METABRIC. We found that tumors with a high ROS score significantly enriched expression of Hallmark cell proliferation gene sets; MYC targets $\mathrm{v} 1$ and v2, G2M checkpoint, and E2F targets (Fig. 1A), as well as other pro-cancerous signaling; glycolysis, MTORC1 signaling, PI3K/AKT/MTOR signaling, oxidative phosphorylation, DNA repair, and Unfolded protein response, in both the cohorts consistently (Fig. 1B). To this end, there is a significant association of high ROS score with both cell proliferation and also other pro-cancer signaling and responses in breast cancer. 
Cancer cells were the major source of ROS in tumor microenvironment (TME), and immune-related gene sets significantly enriched to high ROS breast cancer.

To identify which cell type is the major source of ROS in the tumor microenvironment (TME), the score was measured in a single-cell sequence cohort (GSE75688) that differentiate transcriptome from cancer cells, myeloid cells, T cells, B cells or stromal cells. We found that cancer cells are the major source of ROS in TME (Fig. 2A; $p<0.001$ ). Next, we investigated the relationship between ROS and immune response in TME of breast tumor. We found that immune-related gene sets; allograft rejection, interferon (IFN)- $\gamma$ response, IFN-a response, inflammatory response, complement, and IL6/JAK/STAT3 signaling, were significantly enriched to breast cancer with elevated ROS score in both METABRIC and GSE96058 cohorts consistently (Fig. 2B). To this end, immune response was significantly associated with high ROS score in breast cancer.

Immune cells were universelly infiltrated in ROS high tumor immune microenviornment (TIME) where cytolytic activity (CYT) were elevated

Since immune response was enhanced in ROS high tumor, to investigate which type of immune cell is infiltrated in breast cancer TIME was of interest. The xCell algorithm was used to estimate immune cell infiltration, in a similar fashion as previous publications [36, 39, 44-48]. We found that in high ROS breast cancer, there was significant infiltration of not only pro-cancer immune cells; M2 macrophages, T helper type 2 (Th2) cells, and regulatory T cells, but also anti-cancer immune cells; $\mathrm{CD}^{+}{ }^{+}$memory $\mathrm{T}$ cells, $\mathrm{CD}^{+} \mathrm{T}$ cells, $\mathrm{T}$ helper type 1 (Th1) cells, M1 macrophages, and dendritic cells (DC), and B cells, consistently in both GSE96058 and METABRIC cohorts (Fig. 3A; all $p<0.001$ ). Furthermore, they were significantly associated with high CYT (Fig. 3B; both $p<0.001$ ). To this end, immune cells are universally infiltrated with high cytolytic activity in high ROS breast cancer.

High ROS score was significantly associated with high level of intratumor heterogeneity, homologous recombination deficiency (HRD), and mutation load in breast cancer

Tumor-infiltrating immune cells are known to be attracted to TME through neoantigens generated by high tumor mutational burden [11]. To this end, investigating the relationship of the ROS score with the intratumor heterogeneity, HRD, and mutation load in breast cancer was of interest. We found that breast cancer with a high ROS score was significantly associated with HRD and intratumor heterogeneity, as well as mutation load-related score; single nucleotide variation (SNV) neoantigens, silent and non-silent mutation rates, in the TCGA cohort (Fig. 4; all $p<0.001$ ). This result is in agreement with the notion that immune cells are attracted to cancer cells with high mutation load, and our observation that high ROS score breast cancer is associated with immune response and immune cell infiltration, commonly seen in aggressive cancer.

\section{A high ROS breast cancer was significantly associated with clinical aggressiveness}


To study the clinical relevance of the ROS score in breast cancer, its association with breast cancer subtypes, AJCC (American Joint Committee on Cancer) staging including the lymph node category, the Nottingham histological grade were investigated in the GSE96058 and METABRIC cohorts. In the METABRIC cohort, the high score was significantly associated with triple-negative breast cancer (TNBC) subtype, advanced stage and lymph node metastasis, and higher grade (Fig. 5 , all $p<0.001$ ). GSE96058 cohort was used to validate these results (all $p<0.001$ ). Therefore, ROS score was associated with clinical parameters of tumor aggressiveness.

High ROS ER-positive/HER2-negative breast cancer was significantly associated with worse survival, but not the other subtypes

Given that ROS score was significantly different by breast cancer subtypes, we hypothesized that there may be a different relationship of each subtype with ROS. Therefore, overall survival (OS) in the GSE96058 and METABRIC cohorts and disease specific survival (DSS) in the METABRIC by subtypes were analyzed. High ROS score was significantly associated with worse OS in ER-positive/HER2-negative subtype and the whole group, but not in the other subtypes in the GSE96058 cohort (Fig. 6). OS and DSS in METABRIC cohort validated these results. To this end, high ROS was associated with worse survival outcome only in ER-positive/HER2-negative breast cancer subtype.

\section{Discussion}

In this study, we generated a ROS score utilizing Hallmark ROS pathway gene sets by GSVA algorithm and quantified the abundance of ROS in total of 6,245 breast cancer patients. We found that high ROS breast cancer enriched cell proliferation gene sets; MYC targets $v 1$ and $v 2, G 2 M$ checkpoint, E2F targets, as well as other pro-cancerous gene sets; glycolysis, PI3K/AKT/MTOR signaling, DNA repair, unfolded protein response, MTORC1 signaling, and oxidative phosphorylation, consistently in both GSE96058 and METABRIC cohorts. Cancer cells were the major source of ROS among the cells in TIME, including myeloid cells, T cells, B cells, and stromal cells, based on single cell sequence cohort. High ROS breast cancer enriched immune gene sets; inflammatory response, IL6/JAK/STAT3 signaling, IFN-y response, IFN-a response, allograft rejection, and complement, in both the cohorts consistently. High ROS breast cancer was significantly associated with high infiltration of pro-cancer immune cells; Tregs, Th2 cells, and M2 macrophages, as well as anti-cancer immune cells; M1 macrophages, CD4 ${ }^{+}$memory T cells, CD ${ }^{+} \mathrm{T}$ cells, dendritic cells, Th1 cells, and B-cells in both cohorts. Furthermore, high ROS breast cancer was significantly associated with enhanced CYT. High ROS score was significantly associated with high levels of homologous recombination defects and intratumor heterogeneity, along with mutation-related score, including single nucleotide variant and indel neoantigens, fraction altered, silent and non-silent mutation rate in the TCGA cohort. Clinically, high ROS breast cancer was significantly associated with advanced cancer by AJCC stage, lymph node metastasis, and Nottingham grade. Furthermore, high ROS was significantly associated with worse OS in both cohorts, and DSS in the METABRIC cohort in ERpositive/HER2-negative subtype. 
ROS attack DNA inducing DNA damage which, could either inhibit or induce transcription, replication errors, signal transduction pathways and genomic instability, resulting in carcinogenesis [49]. Several risk factors of breast cancer are related to ROS production and oxidative stress, thus, supporting the role of ROS involvement in breast cancer. Aging is associated with the production of ROS, like $\mathrm{H}_{2} \mathrm{O}_{2}$ and $\mathrm{HOCl}$, that induce mutations in mitochondrial DNA [50]. Mutations in BRCA1 and BRCA2, the breast cancer susceptibility genes, are also implicated in breast cancer. $B R C A 1$ upregulates the expression of multiple genes that are involved in the cytoprotective antioxidant response, such as, glutathione-S-transferase (GST), oxidoreductase, glutathione peroxidase, and other antioxidant genes. A potential source of ROS, ionizing radiation, can induce irreversible DNA damage, thus initiating carcinogenesis. Female breast tissue is particularly susceptible to the carcinogenic effects of ionizing radiation, especially before it is fully differentiated before first child birth [51]. Estrogens are known key player in all stages of breast cancer, where estrogen receptor (ER) independent effects result from oxidative metabolism of estradiol to genotoxic metabolites, which then cause direct damage to DNA [52]. This supports our observed finding that ROS was associated with aggressive breast cancer, as it was associated with higher stage and pathological grade.

We also observed that ROS was associated with higher mutation load in tumors. It is known that ROS can cause unpaired or misrepaired DNA damage in cellular division leading to mutations. ROS induce mutations that commonly causing $\mathrm{G}$ to $\mathrm{T}$ transversions [53], which are the most frequent mutations in the human tumors in the p53 suppressor gene [54]. The presence of oxidative DNA damage in cancer supports the fact that initiation of cancer may at least partly be triggered by ROS [55]. In our study, tumors with high ROS were shown to be enriched for cell proliferation-related gene sets. This is consistent with previous reports that ROS in high amounts is associated with uncontrolled cell proliferation [4, 5]. ROS have also been shown to modulate ubiquitination and phosphorylation of cell cycle associated enzymes. Additionally, growth factor receptors have also been shown to be activated by ROS in the absence of growth factor ligands, thus, controlling cell proliferation. For example, Epidermal Growth Factor Receptor (EGFR) is a receptor tyrosine kinase (RTK) that has been shown to be involved in regulating cell proliferation, survival, migration and differentiation [56]. Cancer tissues have upregulated EGFR expression [57-60]. EGFR signaling is affected by ROS by inhibition of EGFR internalization, thus increasing the receptor's signaling potential [61].

Our study showed that all the immune cells were higher in ROS high breast cancers. Proper T cell activation, proliferation and differentiation depends on low levels of ROS. Tregs are able to persist in the oxidant environment, and therefore, a greater number of Tregs have been detected at tumor sites. Tregs have high antioxidant capacity, and are therefore, less sensitive to oxidant stress-induced cell death. In breast cancer models, ROS were essential for tumor associated macrophages (TAMs) to invade the TIME and acquire a M2 pro-tumorigenic phenotype. ROS also results in impairment of antigen presentation by dendritic cells. However, we observed higher M1 macrophages as well. This could be explained by a general inflammatory state induced by the ROS in the TIME [62]. Our result that cytolytic activity was 
elevated in ROS high tumor agrees with a recent report that suggested that ROS contribute to immunogenic cell death and T-cell mediated immunity [63].

There were several limitations in our study. The degree of ROS signaling was determined by GSVA algorithm with hallmark collection in MSigDB, that may or may not include all the genes related to ROS pathway. Since this is a retrospective study that utilized multiple publicly available large patient cohorts, we did not have access to some of the pertinent clinical data including the details on systemic treatments received and it is assumed that all the patients received "Standard of Care". Our results are based on tumor transcriptomics alone and we are incapable of generating any data on mechanistic role of ROS activity. The novelty of our study is to assess the role of ROS in the TIME using a score combining multiple genes. A prospective trial is needed to confirm the utility of ROS score as a predictive biomarker in breast cancer.

\section{Conclusions}

In conclusion, ROS abundance is associated with cancer aggressiveness, enhanced immune response, and with worse survival in ER-positive/HER2-negative breast cancer.

\section{Abbreviations}

\begin{tabular}{ll} 
AJCC & American Joint Committee on Cancer \\
\hline DFS & disease-free survival \\
\hline DSS & disease-specific survival \\
\hline ER & estrogen receptor \\
\hline FDR & false discovery rate \\
\hline GSEA & Gene set enrichment analysis \\
\hline GSVA & Gene set variation analysis \\
\hline HER2 & human epidermal growth factor receptor 2 \\
\hline METABRIC & Molecular Taxonomy of Breast Cancer International Consortium \\
\hline NES & normalized enrichment score \\
\hline OS & overall survival \\
\hline TNBC & triple negative breast cancer
\end{tabular}

\section{Declarations}

Funding: This research was supported by National Institutes of Health, USA grant number R37CA248018, R01CA250412, R01CA251545, R01EB029596, as well as US Department of Defense BCRP 
grant number W81XWH-19-1-0674 and W81XWH-19-1-0111 to K.T. National Cancer Institute, cancer center support grant P30CA016056 supports Roswell Park Comprehensive Cancer Center. Research reported in this publication was supported by the National Center for Advancing Translational Sciences of the National Institutes of Health under award numbers KL2TR001413 and UL1TR001412. The content is solely the responsibility of the authors and does not necessarily represent the official views of the NIH.

Competing Interests: The authors have no relevant financial or non-financial interests to disclose.

Author Contributions: Conceptualization was performed by Akimitsu Yamada, Itaru Endo, Kazuaki Takabe, Masanori Oshi, and Ryusei Matsuyama. Methodology was performed by Kazuaki Takabe, Masanori Oshi, Yoshihisa Tokumaru. Formal Analysis was performed by Masanori Oshi. The first draft of the manuscript was written by Masanori Oshi, and all authors commented on previous versions of the manuscript. Supervision and project administration were performed by Kazuaki Takabe. All authors read and approved the final manuscript.

Data availability: The datasets generated during and analyzed during the current study are available from the original source as they are publicly available deidentified databases.

Ethics approval: Institutional review board (IRB) approval at Roswell Park Comprehensive Cancer Center (Buffalo, New York, United States of America) was waived as publicly available deidentified databases were used.

Consent to participate: Not applicable.

Consent for publication: Not applicable.

\section{References}

1. Z. Zhou, K. Ni, H. Deng, X. Chen, Dancing with reactive oxygen species generation and elimination in nanotheranostics for disease treatment, Adv Drug Deliv Rev, 158 (2020) 73-90.

2. M. Schieber, N.S. Chandel, ROS function in redox signaling and oxidative stress, Curr Biol, 24 (2014) R453-462.

3. M.L. Circu, T.Y. Aw, Reactive oxygen species, cellular redox systems, and apoptosis, Free Radic Biol Med, 48 (2010) 749-762.

4. P.D. Ray, B.W. Huang, Y. Tsuji, Reactive oxygen species (ROS) homeostasis and redox regulation in cellular signaling, Cell Signal, 24 (2012) 981-990.

5. T. Jenkins, J. Gouge, Nrf2 in Cancer, Detoxifying Enzymes and Cell Death Programs, Antioxidants (Basel), 10 (2021).

6. A.C. Kimmelman, The dynamic nature of autophagy in cancer, Genes Dev, 25 (2011) 1999-2010.

7. T. Shimi, V. Butin-Israeli, S.A. Adam, R.B. Hamanaka, A.E. Goldman, C.A. Lucas, D.K. Shumaker, S.T. Kosak, N.S. Chandel, R.D. Goldman, The role of nuclear lamin B1 in cell proliferation and senescence, 
Genes Dev, 25 (2011) 2579-2593.

8. F. Martinon, Signaling by ROS drives inflammasome activation, Eur J Immunol, 40 (2010) 616-619.

9. J. Tschopp, K. Schroder, NLRP3 inflammasome activation: The convergence of multiple signalling pathways on ROS production?, Nat Rev Immunol, 10 (2010) 210-215.

10. M.G. Vander Heiden, L.C. Cantley, C.B. Thompson, Understanding the Warburg effect: the metabolic requirements of cell proliferation, Science (New York, N.Y.), 324 (2009) 1029-1033.

11. H. Takahashi, M. Asaoka, L. Yan, O.M. Rashid, M. Oshi, T. Ishikawa, M. Nagahashi, K. Takabe, Biologically Aggressive Phenotype and Anti-cancer Immunity Counterbalance in Breast Cancer with High Mutation Rate, Scientific reports, 10 (2020) 1852.

12. A. Liberzon, C. Birger, H. Thorvaldsdóttir, M. Ghandi, J.P. Mesirov, P. Tamayo, The Molecular Signatures Database (MSigDB) hallmark gene set collection, Cell systems, 1 (2015) 417-425.

13. M. Oshi, S. Newman, Y. Tokumaru, L. Yan, R. Matsuyama, I. Endo, M.H.G. Katz, K. Takabe, High G2M Pathway Score Pancreatic Cancer is Associated with Worse Survival, Particularly after MarginPositive (R1 or R2) Resection, Cancers, 12 (2020).

14. M. Oshi, A. Patel, L. Le, Y. Tokumaru, L. Yan, R. Matsuyama, I. Endo, K. Takabe, G2M checkpoint pathway alone is associated with drug response and survival among cell proliferation-related pathways in pancreatic cancer, American journal of cancer research, 11 (2021) 3070-3084.

15. M. Oshi, V. Satyananda, F.A. Angarita, T.H. Kim, Y. Tokumaru, L. Yan, R. Matsuyama, I. Endo, M. Nagahashi, K. Takabe, Angiogenesis is associated with an attenuated tumor microenvironment, aggressive biology, and worse survival in gastric cancer patients, American journal of cancer research, 11 (2021) 1659-1671.

16. M. Oshi, T.H. Kim, Y. Tokumaru, L. Yan, R. Matsuyama, I. Endo, L. Cherkassky, K. Takabe, Enhanced DNA Repair Pathway is Associated with Cell Proliferation and Worse Survival in Hepatocellular Carcinoma (HCC), Cancers, 13 (2021).

17. A. Patel, M. Oshi, L. Yan, R. Matsuyama, I. Endo, K. Takabe, The Unfolded Protein Response Is Associated with Cancer Proliferation and Worse Survival in Hepatocellular Carcinoma, Cancers, 13 (2021).

18. M. Oshi, Y. Tokumaru, F.A. Angarita, L. Yan, R. Matsuyama, I. Endo, K. Takabe, Degree of Early Estrogen Response Predict Survival after Endocrine Therapy in Primary and Metastatic ER-Positive Breast Cancer, Cancers, 12 (2020).

19. Y. Tokumaru, M. Oshi, E. Katsuta, L. Yan, V. Satyananda, N. Matsuhashi, M. Futamura, Y. Akao, K. Yoshida, K. Takabe, KRAS signaling enriched triple negative breast cancer is associated with favorable tumor immune microenvironment and better survival, American journal of cancer research, 10 (2020) 897-907.

20. M. Oshi, S. Newman, Y. Tokumaru, L. Yan, R. Matsuyama, I. Endo, M. Nagahashi, K. Takabe, IntraTumoral Angiogenesis Is Associated with Inflammation, Immune Reaction and Metastatic Recurrence in Breast Cancer, International journal of molecular sciences, 21 (2020). 
21. M. Oshi, S. Newman, Y. Tokumaru, L. Yan, R. Matsuyama, I. Endo, K. Takabe, Inflammation Is Associated with Worse Outcome in the Whole Cohort but with Better Outcome in Triple-Negative Subtype of Breast Cancer Patients, J Immunol Res, 2020 (2020) 5618786.

22. S. Gandhi, M. Oshi, V. Murthy, E.A. Repasky, K. Takabe, Enhanced Thermogenesis in Triple-Negative Breast Cancer Is Associated with Pro-Tumor Immune Microenvironment, Cancers, 13 (2021).

23. M. Oshi, Y. Tokumaru, F.A. Angarita, L. Lee, L. Yan, R. Matsuyama, I. Endo, K. Takabe, Adipogenesis in triple-negative breast cancer is associated with unfavorable tumor immune microenvironment and with worse survival, Scientific reports, 11 (2021) 12541.

24. V. Murthy, M. Oshi, Y. Tokumaru, I. Endo, K. Takabe, Increased apoptosis is associated with robust immune cell infiltration and cytolytic activity in breast cancer, American journal of cancer research, 11 (2021) 3674-3687.

25. M. Oshi, H. Takahashi, Y. Tokumaru, L. Yan, O.M. Rashid, R. Matsuyama, I. Endo, K. Takabe, G2M Cell Cycle Pathway Score as a Prognostic Biomarker of Metastasis in Estrogen Receptor (ER)-Positive Breast Cancer, International journal of molecular sciences, 21 (2020).

26. M. Oshi, H. Takahashi, Y. Tokumaru, L. Yan, O.M. Rashid, M. Nagahashi, R. Matsuyama, I. Endo, K. Takabe, The E2F Pathway Score as a Predictive Biomarker of Response to Neoadjuvant Therapy in ER+/HER2- Breast Cancer, Cells, 9 (2020).

27. O.M. Rueda, S.J. Sammut, J.A. Seoane, S.F. Chin, J.L. Caswell-Jin, M. Callari, R. Batra, B. Pereira, A. Bruna, H.R. Ali, E. Provenzano, B. Liu, M. Parisien, C. Gillett, S. McKinney, A.R. Green, L. Murphy, A. Purushotham, I.O. Ellis, P.D. Pharoah, C. Rueda, S. Aparicio, C. Caldas, C. Curtis, Dynamics of breastcancer relapse reveal late-recurring ER-positive genomic subgroups, Nature, 567 (2019) 399-404.

28. C. Curtis, S.P. Shah, S.F. Chin, G. Turashvili, O.M. Rueda, M.J. Dunning, D. Speed, A.G. Lynch, S. Samarajiwa, Y. Yuan, S. Gräf, G. Ha, G. Haffari, A. Bashashati, R. Russell, S. McKinney, A. Langerød, A. Green, E. Provenzano, G. Wishart, S. Pinder, P. Watson, F. Markowetz, L. Murphy, I. Ellis, A. Purushotham, A.L. Børresen-Dale, J.D. Brenton, S. Tavaré, C. Caldas, S. Aparicio, The genomic and transcriptomic architecture of 2,000 breast tumours reveals novel subgroups, Nature, 486 (2012) 346-352.

29. C. Brueffer, S. Gladchuk, C. Winter, J. Vallon-Christersson, C. Hegardt, J. Häkkinen, A.M. George, Y. Chen, A. Ehinger, C. Larsson, N. Loman, M. Malmberg, L. Rydén, Å. Borg, L.H. Saal, The mutational landscape of the SCAN-B real-world primary breast cancer transcriptome, EMBO molecular medicine, 12 (2020) e12118.

30. J. Liu, T. Lichtenberg, K.A. Hoadley, L.M. Poisson, A.J. Lazar, A.D. Cherniack, A.J. Kovatich, C.C. Benz, D.A. Levine, A.V. Lee, L. Omberg, D.M. Wolf, C.D. Shriver, V. Thorsson, H. Hu, An Integrated TCGA PanCancer Clinical Data Resource to Drive High-Quality Survival Outcome Analytics, Cell, 173 (2018) 400-416.e411.

31. W. Chung, H.H. Eum, H.O. Lee, K.M. Lee, H.B. Lee, K.T. Kim, H.S. Ryu, S. Kim, J.E. Lee, Y.H. Park, Z. Kan, W. Han, W.Y. Park, Single-cell RNA-seq enables comprehensive tumour and immune cell profiling in primary breast cancer, Nature communications, 8 (2017) 15081. 
32. S. Hänzelmann, R. Castelo, J. Guinney, GSVA: gene set variation analysis for microarray and RNA-seq data, BMC bioinformatics, 14 (2013) 7.

33. S. Narayanan, T. Kawaguchi, L. Yan, X. Peng, Q. Qi, K. Takabe, Cytolytic Activity Score to Assess Anticancer Immunity in Colorectal Cancer, Annals of surgical oncology, 25 (2018) 2323-2331.

34. T. Takeshita, T. Torigoe, L. Yan, J.L. Huang, H. Yamashita, K. Takabe, The Impact of Immunofunctional Phenotyping on the Malfunction of the Cancer Immunity Cycle in Breast Cancer, Cancers, 13 (2020).

35. M. Oshi, T. Kawaguchi, L. Yan, X. Peng, Q. Qi, W. Tian, A. Schulze, K.A. McDonald, S. Narayanan, J. Young, S. Liu, L.G. Morris, T.A. Chan, P. Kalinski, R. Matsuyama, E. Otsuji, I. Endo, K. Takabe, Immune cytolytic activity is associated with reduced intra-tumoral genetic heterogeneity and with better clinical outcomes in triple negative breast cancer, American journal of cancer research, 11 (2021) 3628-3644.

36. M. Oshi, S. Gandhi, M.R. Huyser, Y. Tokumaru, L. Yan, A. Yamada, R. Matsuyama, I. Endo, K. Takabe, MELK expression in breast cancer is associated with infiltration of immune cell and pathological compete response (pCR) after neoadjuvant chemotherapy, American journal of cancer research, 11 (2021) 4421-4437.

37. M.S. Rooney, S.A. Shukla, C.J. Wu, G. Getz, N. Hacohen, Molecular and genetic properties of tumors associated with local immune cytolytic activity, Cell, 160 (2015) 48-61.

38. D. Aran, Z. Hu, A.J. Butte, xCell: digitally portraying the tissue cellular heterogeneity landscape, Genome biology, 18 (2017) 220.

39. M. Oshi, M. Asaoka, Y. Tokumaru, F.A. Angarita, L. Yan, R. Matsuyama, E. Zsiros, T. Ishikawa, I. Endo, K. Takabe, Abundance of Regulatory T Cell (Treg) as a Predictive Biomarker for Neoadjuvant Chemotherapy in Triple-Negative Breast Cancer, Cancers, 12 (2020).

40. M. Oshi, S. Newman, V. Murthy, Y. Tokumaru, L. Yan, R. Matsuyama, I. Endo, K. Takabe, ITPKC as a Prognostic and Predictive Biomarker of Neoadjuvant Chemotherapy for Triple Negative Breast Cancer, Cancers, 12 (2020).

41. M. Oshi, Y. Tokumaru, A. Patel, L. Yan, R. Matsuyama, I. Endo, M.H.G. Katz, K. Takabe, A Novel FourGene Score to Predict Pathologically Complete (R0) Resection and Survival in Pancreatic Cancer, Cancers, 12 (2020).

42. V. Thorsson, D.L. Gibbs, S.D. Brown, D. Wolf, D.S. Bortone, T.H. Ou Yang, E. Porta-Pardo, G.F. Gao, C.L. Plaisier, J.A. Eddy, E. Ziv, A.C. Culhane, E.O. Paull, I.K.A. Sivakumar, A.J. Gentles, R. Malhotra, F. Farshidfar, A. Colaprico, J.S. Parker, L.E. Mose, N.S. Vo, J. Liu, Y. Liu, J. Rader, V. Dhankani, S.M. Reynolds, R. Bowlby, A. Califano, A.D. Cherniack, D. Anastassiou, D. Bedognetti, Y. Mokrab, A.M. Newman, A. Rao, K. Chen, A. Krasnitz, H. Hu, T.M. Malta, H. Noushmehr, C.S. Pedamallu, S. Bullman, A.I. Ojesina, A. Lamb, W. Zhou, H. Shen, T.K. Choueiri, J.N. Weinstein, J. Guinney, J. Saltz, R.A. Holt, C.S. Rabkin, A.J. Lazar, J.S. Serody, E.G. Demicco, M.L. Disis, B.G. Vincent, I. Shmulevich, The Immune Landscape of Cancer, Immunity, 51 (2019) 411-412. 
43. A. Subramanian, P. Tamayo, V.K. Mootha, S. Mukherjee, B.L. Ebert, M.A. Gillette, A. Paulovich, S.L. Pomeroy, T.R. Golub, E.S. Lander, J.P. Mesirov, Gene set enrichment analysis: a knowledge-based approach for interpreting genome-wide expression profiles, Proceedings of the National Academy of Sciences of the United States of America, 102 (2005) 15545-15550.

44. V. Satyananda, M. Oshi, Y. Tokumaru, A. Maiti, N. Hait, R. Matsuyama, I. Endo, K. Takabe, Sphingosine 1-phosphate (S1P) produced by sphingosine kinase 1 (SphK1) and exported via ABCC1 is related to hepatocellular carcinoma (HCC) progression, American journal of cancer research, 11 (2021) 4394-4407.

45. E. Katsuta, M. Huyser, L. Yan, K. Takabe, A prognostic score based on long-term survivor unique transcriptomic signatures predicts patient survival in pancreatic ductal adenocarcinoma, American journal of cancer research, 11 (2021) 4294-4307.

46. M. Oshi, S. Gandhi, F.A. Angarita, T.H. Kim, Y. Tokumaru, L. Yan, R. Matsuyama, I. Endo, K. Takabe, A novel five-gene score to predict complete pathological response to neoadjuvant chemotherapy in ERpositive/HER2-negative breast cancer, American journal of cancer research, 11 (2021) 3611-3627.

47. M. Oshi, S. Newman, Y. Tokumaru, L. Yan, R. Matsuyama, P. Kalinski, I. Endo, K. Takabe, Plasmacytoid Dendritic Cell (pDC) Infiltration Correlate with Tumor Infiltrating Lymphocytes, Cancer Immunity, and Better Survival in Triple Negative Breast Cancer (TNBC) More Strongly than Conventional Dendritic Cell (cDC), Cancers, 12 (2020).

48. M. Oshi, M. Asaoka, Y. Tokumaru, L. Yan, R. Matsuyama, T. Ishikawa, I. Endo, K. Takabe, CD8 T Cell Score as a Prognostic Biomarker for Triple Negative Breast Cancer, International journal of molecular sciences, 21 (2020).

49. M. Valko, C.J. Rhodes, J. Moncol, M. Izakovic, M. Mazur, Free radicals, metals and antioxidants in oxidative stress-induced cancer, Chem Biol Interact, 160 (2006) 1-40.

50. D. Harman, Free radical theory of aging: an update: increasing the functional life span, Ann N Y Acad Sci, 1067 (2006) 10-21.

51. D.L. Preston, A. Mattsson, E. Holmberg, R. Shore, N.G. Hildreth, J.D. Boice, Jr., Radiation effects on breast cancer risk: a pooled analysis of eight cohorts, Radiat Res, 158 (2002) 220-235.

52. W. Yue, R.J. Santen, J.P. Wang, Y. Li, M.F. Verderame, W.P. Bocchinfuso, K.S. Korach, P. Devanesan, R. Todorovic, E.G. Rogan, E.L. Cavalieri, Genotoxic metabolites of estradiol in breast: potential mechanism of estradiol induced carcinogenesis, J Steroid Biochem Mol Biol, 86 (2003) 477-486.

53. K.G. Higinbotham, J.M. Rice, B.A. Diwan, K.S. Kasprzak, C.D. Reed, A.O. Perantoni, GGT to GTT transversions in codon 12 of the K-ras oncogene in rat renal sarcomas induced with nickel subsulfide or nickel subsulfide/iron are consistent with oxidative damage to DNA, Cancer research, 52 (1992) 4747-4751.

54. D.E. Brash, J.A. Rudolph, J.A. Simon, A. Lin, G.J. McKenna, H.P. Baden, A.J. Halperin, J. Pontén, A role for sunlight in skin cancer: UV-induced p53 mutations in squamous cell carcinoma, Proceedings of the National Academy of Sciences of the United States of America, 88 (1991) 10124-10128. 
55. H.E. Poulsen, H. Prieme, S. Loft, Role of oxidative DNA damage in cancer initiation and promotion, Eur J Cancer Prev, 7 (1998) 9-16.

56. H. Masuda, D. Zhang, C. Bartholomeusz, H. Doihara, G.N. Hortobagyi, N.T. Ueno, Role of epidermal growth factor receptor in breast cancer, Breast Cancer Res Treat, 136 (2012) 331-345.

57. J.P. Spano, C. Lagorce, D. Atlan, G. Milano, J. Domont, R. Benamouzig, A. Attar, J. Benichou, A. Martin, J.F. Morere, M. Raphael, F. Penault-Llorca, J.L. Breau, R. Fagard, D. Khayat, P. Wind, Impact of EGFR expression on colorectal cancer patient prognosis and survival, Ann Oncol, 16 (2005) 102-108.

58. G.E. Konecny, L. Santos, B. Winterhoff, M. Hatmal, G.L. Keeney, A. Mariani, M. Jones, C. Neuper, B. Thomas, L. Muderspach, D. Riehle, H.J. Wang, S. Dowdy, K.C. Podratz, M.F. Press, HER2 gene amplification and EGFR expression in a large cohort of surgically staged patients with nonendometrioid (type II) endometrial cancer, British journal of cancer, 100 (2009) 89-95.

59. P.A. Jänne, J.C. Yang, D.W. Kim, D. Planchard, Y. Ohe, S.S. Ramalingam, M.J. Ahn, S.W. Kim, W.C. Su, L. Horn, D. Haggstrom, E. Felip, J.H. Kim, P. Frewer, M. Cantarini, K.H. Brown, P.A. Dickinson, S. Ghiorghiu, M. Ranson, AZD9291 in EGFR inhibitor-resistant non-small-cell lung cancer, N Engl J Med, 372 (2015) 1689-1699.

60. M. Oshi, S. Gandhi, Y. Tokumaru, L. Yan, A. Yamada, R. Matsuyama, T. Ishikawa, I. Endo, K. Takabe, Conflicting roles of EGFR expression by subtypes in breast cancer, American journal of cancer research, 11 (2021) 5094-5110.

61. E.H. Verbon, J.A. Post, J. Boonstra, The influence of reactive oxygen species on cell cycle progression in mammalian cells, Gene, 511 (2012) 1-6.

62. A. Kotsafti, M. Scarpa, I. Castagliuolo, M. Scarpa, Reactive Oxygen Species and Antitumor ImmunityFrom Surveillance to Evasion, Cancers, 12 (2020).

63. S. Guo, N.I. Burcus, M. Scott, Y. Jing, I. Semenov, The role of reactive oxygen species in the immunity induced by nano-pulse stimulation, Scientific reports, 11 (2021) 23745.

\section{Supplementary Tables}

Table S1 is not available with this version

\section{Figures}

\section{Figure 1}

Cell proliferation-related and other pro-cancer signaling-related gene sets enriched to breast cancer with a high ROS score consistently in both GSE96058 and METABRIC cohorts

a. Enrichment plots of cell proliferation-related gene sets (MYC targets v1 and v2, G2M checkpoint, E2F targets), and b. other pro-cancerous gene sets (glycolysis, PI3K/AKT/MTOR signaling, DNA repair, 
Unfolded protein response, oxidative phosphorylation, and MTORC1 signaling) in the GSE96058 and METABRIC cohorts. The classical gene set enrichment analysis (GSEA) method was used to determine the NES (normalized enrichment score) and the FDR (false discovery rate) where the cut-off to divide two score groups was defined as median value

\section{Figure 2}

\section{Relationship between ROS and immune response in breast cancer TME}

a. Boxplots showing ROS by single cells of the cancer, myeloid cells, B cells, T cells, and stromal cells in the GSE75688 cohort. P-values were calculated by the Kruskal-Wallis test. b. Gene enrichment plots of allograft rejection, interferon (IFN)- $\gamma$ response, IFN-a response, inflammatory response, complement, and IL6/JAK/STAT3 signaling, that enriched to high ROS score in both cohorts. The median value was used as a cut-off to divide high vs low score groups. NES (normalized enrichment score) and FDR (false discovery rate) were determined with the classical gene set enrichment analysis (GSEA) method

\section{Figure 3}

\section{Infiltrating immune cells by the ROS score in breast cancer}

Boxplots of infiltrating fraction of a. pro-cancer immune cells; regulatory $\mathrm{T}$ cells (Tregs), type2 $\mathrm{T}$ (Th2) cells, M2 macrophages, and anti-cancer immune cells; $\mathrm{CD} 4^{+}$memory $\mathrm{T}$ cells, $\mathrm{CD} 8^{+} \mathrm{T}$ cells, type $1 \mathrm{~T}$ helper (Th1) cells, M1 macrophages, and B cells by high and low ROS score groups in the GSE96058 and METABRIC cohorts. b. Box plots of cytolytic activity score (CYT) by low and high ROS score groups in both cohorts. The median value was used as a cut-off to divide two score groups. $P$-values were calculated by the Mann-Whitney $\mathrm{U}$ test

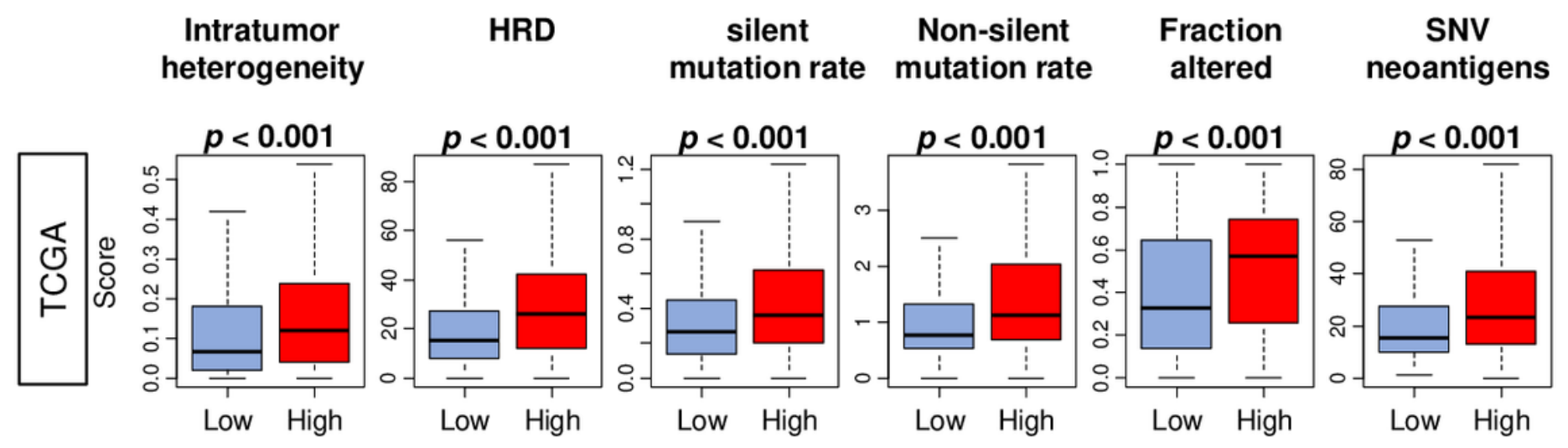


Figure 4

Association of the ROS score with the intratumor heterogeneity, homologous recombination deficiency (HRD), and mutation-related score in the TCGA cohort

Boxplots of homologous recombination defects (HRD), and intratumor heterogeneity, and the mutationrelated scores; silent and non-silent mutation rate, fraction altered, single nucleotide variation (SNV) neoantigens, by low and high ROS score groups. The median value was used as a cut-off to divide two score groups. $P$-values were calculated by the Mann-Whitney $U$ test

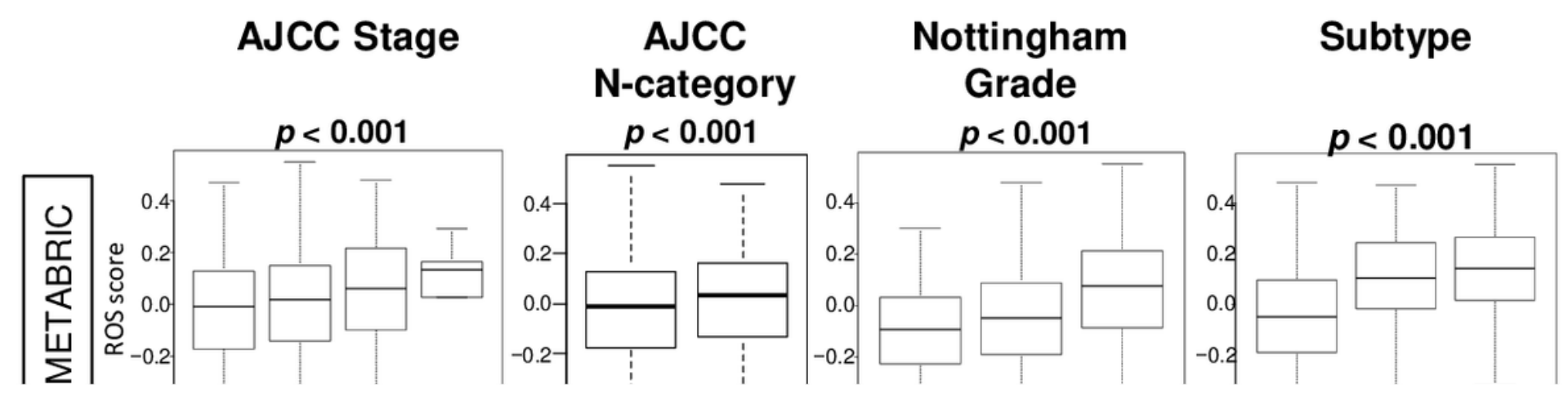

\section{Figure 5}

Breast cancer with a high ROS score was significantly associated with advanced stage, lymph node metastasis, higher pathological grade, and TNBC

Box plots of the score by American Joint Committee on Cancer (AJCC) stage in the METABRIC, and lymph node metastasis status, Nottingham pathological grade, and breast cancer subtypes in the METABRIC and GSE96058 cohorts. P values were calculated by Kruskal-Wallis and Mann-Whitney U test.

Figure 6 
ER-positive/HER2-negative breast cancer with a high score is significantly associated with worse survival in the GSE96058 and METABRIC cohorts

Forest plots with log-rank $p$-values and hazard ratio (HR) of OS in the GSE96058 cohort, and OS and DSS in the METABRIC cohort between low and high ROS score in the whole, ER+/HER2-, HER2+ and TNBC subgroups. Log-rank test was used to calculate $p$ values. DSS, disease-specific survival; ER, estrogen receptor; HER2, human epidermal growth factor 2; OS, overall survival; TNBC, triple-negative breast cancer 